\title{
Prevalence of Decayed Tooth Surfaces in Preschool Children and Factors Influencing its Development: An Observational Study
}

\author{
Vinay Kumar Srivastava ${ }^{1}$
}

'Department of Pedodontics and Preventive Dentistry, Faculty of Dental Sciences, Institute of Medical Sciences, Banaras Hindu University, Varanasi, India

\author{
Address for correspondence: Vinay Kumar Srivastava, BDS, MDS, \\ Pedodontics and Preventive Dentistry, Faculty of Dental Sciences, \\ Institute of Medical Sciences, Banaras Hindu University Campus, \\ Varanasi, Uttar Pradesh 221005, India \\ E-mail: dr.vinaypedo@gmail.com
}

\begin{abstract}
Objective: Objective of the study was to determine prevalence of decayed tooth surfaces and factors influencing its development in primary dentition.

Materials and Methods: The study design adopted was analytical observational study. Caries examination was conducted using plain mouth mirror, probe with WHO caries detection criteria. Decayed tooth surfaces were examined under optimal light source by a single qualified examiner to minimize visual error. The obtained data were subjected to statistical analysis using SPSS 16 software. Test for significance was done with the help of Chi square test.

Results: Highest percentage of decayed tooth surfaces was observed at 5 years of age followed by 6, 4 and 3 years of age. There was slight predilection in development of decayed tooth surfaces in males than females. Higher parental education levels also significantly reduce prevalence of decayed tooth surfaces in preschool children. A significantly higher percentage of upper and upper middle-class children collectively had decayed teeth than of the collective middle class, lower middle class and lower class. The ratio of breast $\&$ bottle feeding to breast feeding habit only was 32.3:67.7. A significant association was observed between decayed tooth surfaces and feeding habits. As duration of the breast feeding and bottle feeding increased, the percentage of decayed tooth surfaces also increased.
\end{abstract}

Conclusion: Among the studied factors; maternal education level, parental social class, feeding habits, duration of breast \& bottle feeding had the most significant effect on the development of decayed tooth surfaces in preschool children.

Keywords: Decayed surfaces, diet, primary teeth, parent's education, socioeconomic status

\section{Introduction}

Children are the future of our country. Healthy children contribute to the development of the nation. In today's scenario, there are many burning national issues regarding children's health. Among the most important health problems are oral health issues and the most common oral disease is dental caries. There is no geographic area in the world whose human inhabitants do not exhibit some evidence of dental caries. It affects all

How to cite this article: Srivastava VK. Prevalence of Decayed Tooth Surfaces in Preschool Children and Factors Influencing its Development: An Observational Study. J Pediatr Dent 2020;6(1):26-32 
gender, socioeconomic and age groups. [1] Dental caries is a disease which may start as soon as the tooth erupts into the oral cavity, depending on many factors like microorganisms, tooth structure and its mineralization, topography of crown, position in the arch, oral hygiene status, as well as primary and secondary factors associated with development of dental caries. Studies have also shown that there is a relationship between dental caries and socioeconomic status.[2,3] The aim of the study is to find out factors influencing development of tooth decay in primary dentition. These factors become significant to the researcher for prevention of dental caries. In a country like India, which has various food cultures and social classes, these factors may also influence the development of decayed tooth. This study reveals the effects of age, gender, parental education level, social class, dietary habits, breakfast habits, feeding methods, and duration of breast, as well as bottle feeding on development of decayed surfaces of primary teeth.

\section{Materials and Methods}

Children $(\mathrm{n}=1800)$ aged between 3-6 years $(56.7 \%$ male and $43.3 \%$ female) were evaluated for decayed tooth surfaces of primary teeth. Ethical clearance was obtained from the institution and signed written consent was obtained from parents of children participating in the study. Exclusion criteria of the study include missing primary teeth, filled primary teeth, erupted permanent teeth, children suffering from systemic diseases, and special care need. Caries examination was conducted using plain mouth mirror, probe and tweezers with cotton pellets to clean the tooth surface before recording data under optimal light source by using WHO caries detection criteria.[4] Each tooth surface was scored independently. No radiographic examination was done. A standard questionnaire was prepared regarding above mentioned variables of the study based on the memory of the mothers. Parental social class of participating children was also recorded as per Modified BG Prasad's socio-economic classification 2018.[5] It is an income-based scale and therefore, constant update is required to take inflation and depreciation of rupees into accounts. Consumer price index (CPI) is used to calculate updated income categories at any given point of time. Data was recorded from the participating children aged between 3 to 6 years who visited Faculty of Dental Sciences, IMS, BHU, Varanasi, India.

Decayed surfaces were noted, and all children were examined under standardized environment by one qualified examiner. Children's ages were measured in years and for those children whose ages were given in years and months, the ages were rounded off to the nearest year.[6]

\section{Statistical analysis}

Data was processed and statistically analyzed by using Chi square test with the help of SPSS version 16 (Statistical Package for Social Sciences, IBM, Chicago, Illinois, USA) software. Chi square test was done to compare the study population variable factors.

\section{Results}

Fig. 1 shows the total percentage of tooth decayed surfaces in preschool children in association with age. The prevalence of carious decayed surfaces was highest at the age of 5 years followed by 6,4 and 3 years. But no significant correlation was observed between decayed primary tooth surfaces and age variables $(\mathrm{p}=0.230)$.

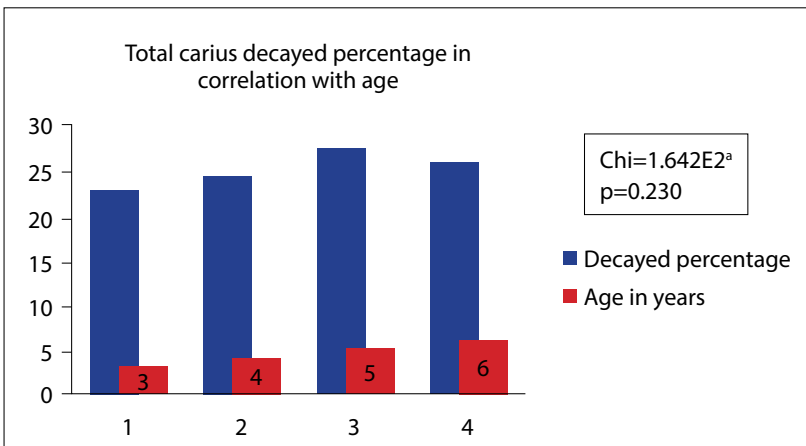

Figure 1. Column diagram of decayed percentages of primary tooth with different age groups of preschool children

Table 1. Percentage of carious decayed surfaces in the study population association with their parental education level

\begin{tabular}{|l|c|c|c|c|c|c|}
\hline Education level & Illiterate & High school & Intermediate & Graduate & Postgraduate & \\
\hline Father education & 2.3 & 10.3 & 28 & 49 & 10.3 & ${\text { Chi }=2.318 \mathrm{BE} 2^{\mathrm{a}}}$ \\
\hline & & & & & $\mathrm{p}=0.790$ \\
\hline Mother education & 7.7 & 12 & 24.3 & 50.3 & 5.7 & $\mathrm{Chi}^{2} 2.356 \mathrm{E} 2^{\mathrm{a}}$ \\
\hline & & & & & $\mathrm{p}=0.043$ \\
\hline
\end{tabular}


Fig. 2 shows gender-wise total percentage of cariously decayed surfaces in preschool children. The prevalence of carious decayed tooth surfaces was slightly more in males than females however no significant correlation was established in gender variables $(\mathrm{p}=0.610)$.

Table 1 reveals percentages of decayed primary tooth surfaces among study population in relation to paternal and maternal education variables. Percentage of decayed tooth surfaces increased from illiterate parent (both mother and father) up to graduate level and abruptly decreased when parental education was post graduate. Paternal education variables have no significant effect on the development of primary tooth decay $(p=0.790)$, while maternal education variables had a significant effect on the development of primary tooth decay $(\mathrm{p}=0.043)$.

Fig. 3 reveals association between percentage of decayed tooth surfaces and parental social class. The percentage of decayed tooth surfaces was highest in upper middle class followed by upper class, middle class, lower middle class and lower class. As per

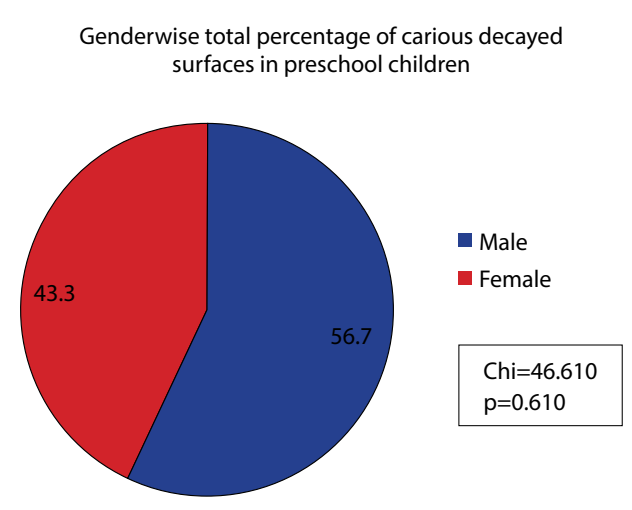

Figure 2. Pie diagram showing Gender wise decayed primary tooth percentages in preschool children

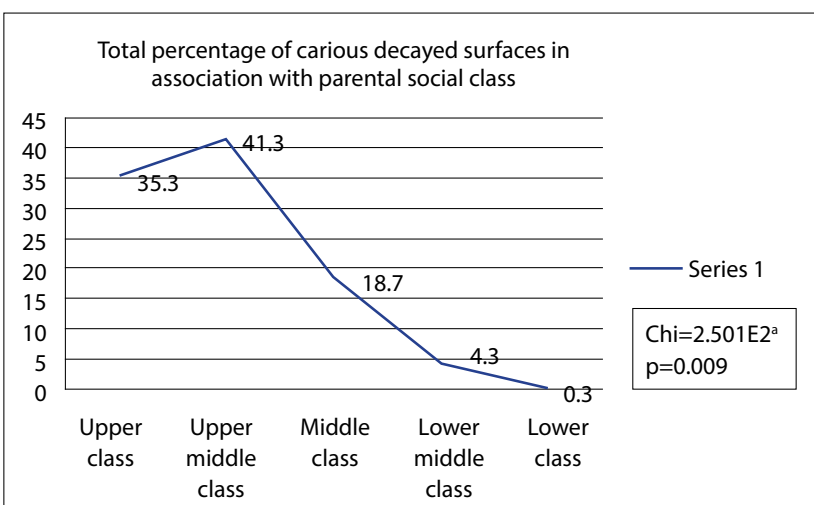

Figure 3. Showed decayed primary tooth percentages of preschool children in association with different parental social class
Prasad's classification of social class among the study population; children of upper and upper middle class collectively had $76.6 \%$ decayed tooth surfaces as compared to the middle class, lower middle class and lower-class collective, which had only $23.3 \%$ decayed tooth surfaces. Thus, it can be inferred that decayed tooth surfaces in primary teeth were in decreasing order from higher class to lower class. Social class of parents had significant effect on the development of tooth decay $(\mathrm{p}=0.009)$.

Fig. 4 reveals association between percentages of decayed primary teeth surfaces with the history of snacks consumption. Among the study population, decayed primary tooth surfaces were $16.3 \%$ in those children who had history of consuming sugary snacks only, and $9.3 \%$ in those children who had history of consuming non-sugary snacks only whereas $74.3 \%$ of decayed surfaces were observed in those children who had history of consumed both sugary and non-sugary snacks. No significant $(p=0.214)$ association was found between decayed surfaces of primary teeth and snack variables. Highest percentage of decayed surfaces was observed in those children who had history of consuming both sugary and non-sugary snack followed by sugary-snack only and non-sugary snack only.

Fig. 5 reveals association between percentages of decayed primary tooth surfaces to vegetarian and nonvegetarian dietary habit. Decayed primary tooth surfaces were observed in $68.7 \%$ of those children who had history of vegetarian diet only, while only in $31.3 \%$ of those children who had history of both vegetarian and non-vegetarian dietary habit, decayed primary tooth surfaces were observed. A higher percentage of children who consumed vegetarian diet as compared to those who consumed both vegetarian and non-vegetarian diet, had decayed tooth surfaces. However, no significant association was observed between cariously

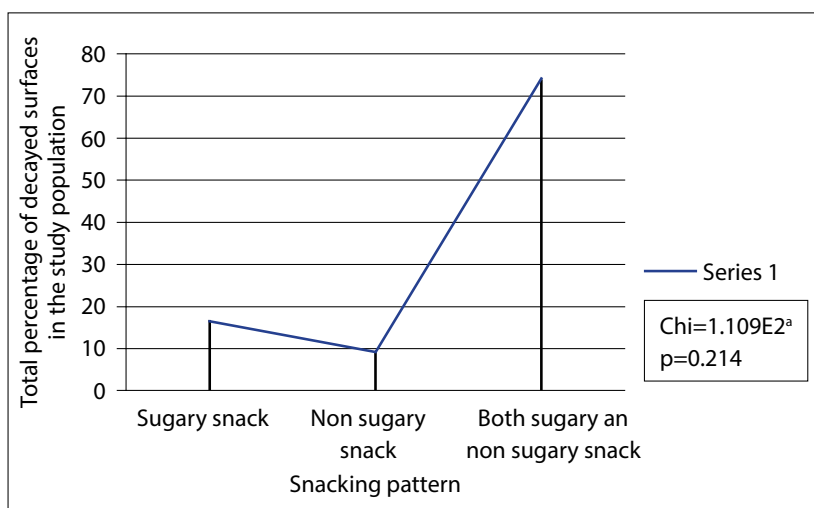

Figure 4. Showed decayed primary tooth percentages of preschool children in association with snacks 


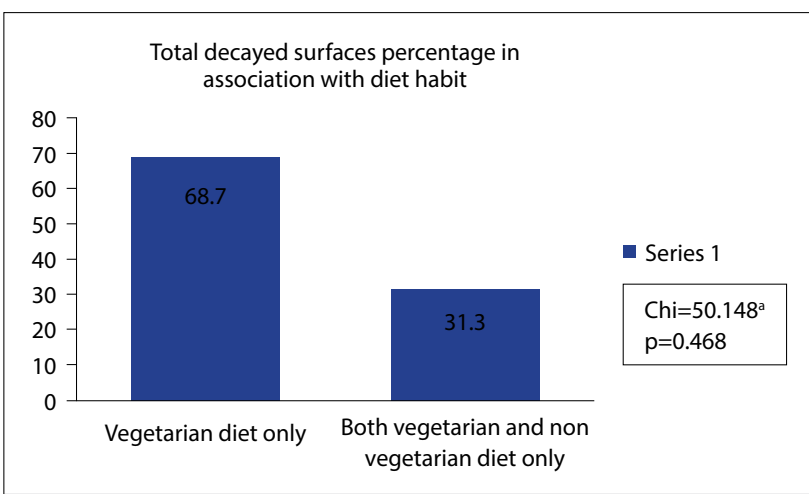

Figure 5. Showed tooth decayed percentages of preschool children in association with dietary habits (vegetarian and nonvegetarian diet)

decayed tooth surfaces to vegetarian/non vegetarian dietary habits $(\mathrm{p}=0.468)$.

Fig. 6 reveals association between decayed primary tooth surfaces and breakfast consumed. Among the study population, Decayed primary tooth surfaces were observed in $67.7 \%$ of those children who had a history of consumption of Roti/Chapatti with Mustard oil and salt (Traditional middle eastern-northern Indian breakfast) or with vegetables, but only in $24 \%$ of those children who consumed another common traditional breakfast in the form of tea with Paratha and only in $8.3 \%$ of those children who had history of consumption of breakfast tea/milk with Cheese- bread/ bread butter. No significant $(\mathrm{p}=0.719)$ association was observed between decayed primary tooth surfaces and breakfast variables.

Fig. 7 reveals associations between percentages of children with decayed primary tooth surfaces and feeding habits. Among the study population, decayed tooth surfaces of primary teeth were observed in $67.7 \%$ of those children who had history of breast-feeding habit only, and in $32.3 \%$ of those children who had history of

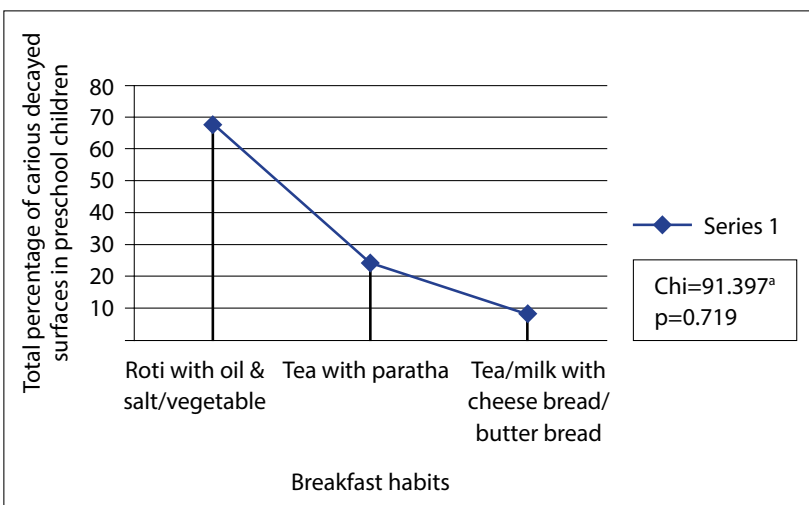

Figure 6. Showed tooth decayed percentages of preschool children in association with breakfast habits

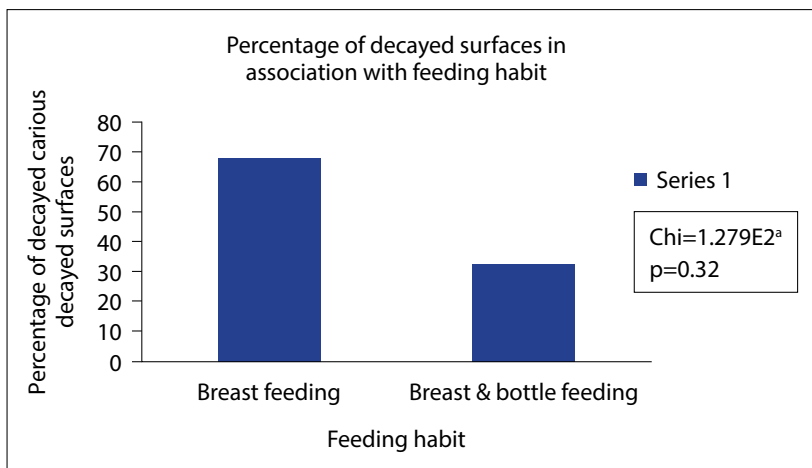

Figure 7. Showed tooth decayed percentages of preschool children in association with breast-and bottle-feeding habits

both breast and bottle feeding. Feeding habit significantly $(\mathrm{p}=0.032)$ affects the development of decayed tooth surfaces.

Fig. 8 reveals association between percentages of children with decayed primary tooth surfaces and duration of breast feeding. Among the study population, decayed primary tooth surfaces were observed in $1.3 \%$ those children who had history of less than 3 months of breast feeding and in $5.3 \%, 17 \%$ and $76.3 \%$ of those children who had history of breast feeding ranging from, 3 to 6 months, 7-12 months and more than 12 months respectively. Thus, there is a significant correlation between decayed primary tooth surfaces and the duration of breast feeding $(\mathrm{p}=0.001)$. As duration of breast feeding increased, chances of cariously decayed surfaces also increased.

Fig. 9 reveals the association between percentages of children with decayed primary tooth surfaces and bottle feeding (day and night) duration. Among the study population, no decayed tooth surfaces were observed in those children who had history of bottle-feeding duration less than 6 months. Decayed tooth surfaces were observed in $3 \%, 12.3 \%, 16.3 \%$ and $68.3 \%$ of those chil-

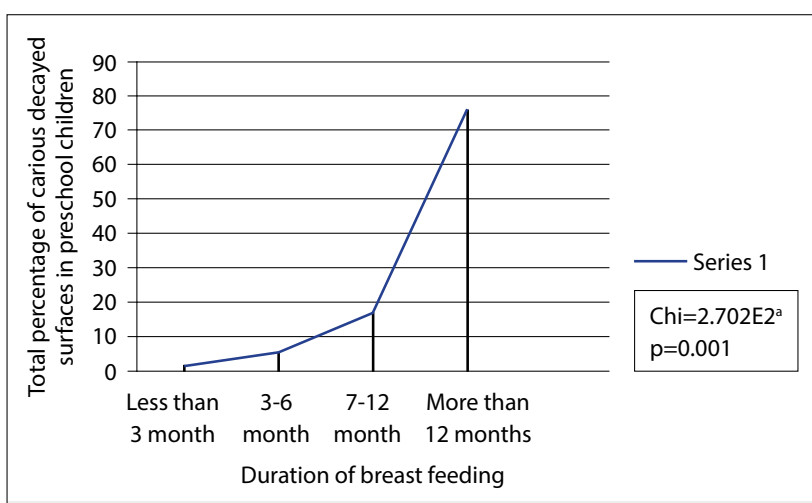

Figure 8. Showed tooth decayed percentages of preschool children in association with duration of breast feeding 


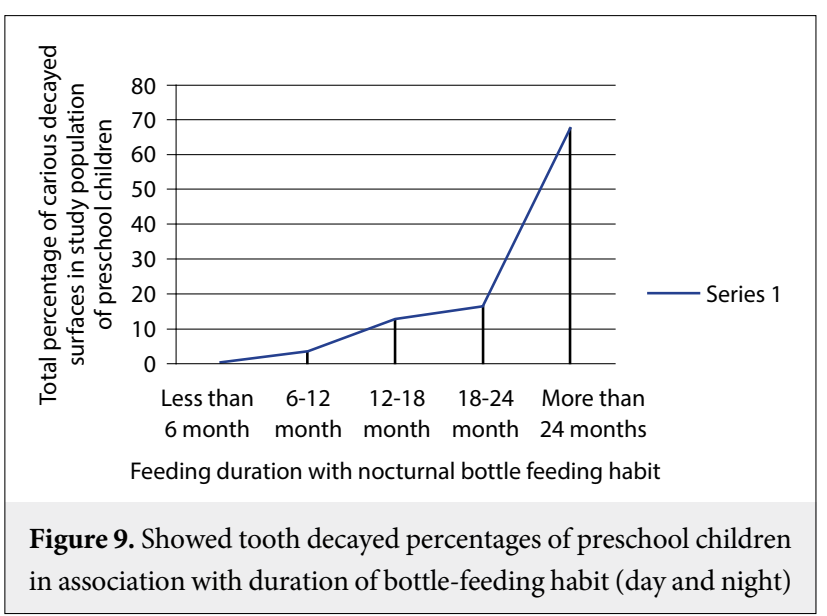

dren who had history of day \& night bottle feeding for the period of 6 to 12 months, 12 to 18 months, 18 to 24 months and more than 24 months respectively. A highly significant $(\mathrm{p}=0.000)$ association observed between bottle feeding duration to decayed tooth primary teeth surfaces.

\section{Discussion}

The results of the present study are based on the prevalence of decayed primary tooth surfaces in preschool children and its association with the history provided by the parent regarding the study variables. Therefore, we cannot rule out the possibility of errors because history is based on the parent's memory (Memory bias).

Children are prone to development of caries. In our study, caries prevalence was $25 \%$ in preschool children. This finding is in accordance with the finding of Vignarajah and Williams [7] and Hattab et al [8] whose study revealed $8.9 \%$ to $36 \%$ caries prevalence. The prevalence of decayed surfaces was highest at the age of 5 years followed by 6,4 and 3 years in preschool children. In our study $56.7 \%$ males and $43.3 \%$ females were suffering from decayed tooth surfaces. Similar trends were reported by Al-Hosani and Rugg-Gunn.[9] There was no significant difference in decayed primary tooth surfaces in preschool children in relation to gender variables $(\mathrm{p}=0.610)$. Thus, it infers that gender is not a risk factor for dental caries.

Parental education levels not only affect living status of the parent but also the living standards of the child. Educated, aware parents become more cautious regarding oral hygiene status and they restrict their children the consumption of carious prone diet or snacks etc. In our study, paternal and maternal education levels were observed along with their children's tooth decay per- centages. When both maternal and paternal education were considered, the percentage of children with cariously decayed surfaces gradually increased from illiterate parents up to graduate level of education but drastically decreased if their education was up to post graduate. It infers that level of education is significant in reducing percentage of preschool children with decayed tooth surfaces.

No significant association was observed between decayed primary tooth surfaces to paternal education variables. However, maternal education variable significantly affected the development of primary tooth decay in preschool children. Higher the level of maternal education lesser will be percentage of tooth decay. This might be because highly educated mothers are more concerned and conscious about oral health of their children as compared to fathers. Thus, a maternal factor in terms of education level seems to have greater impact on cariously dental decay than paternal education. This may be due to the care and nurture usually provided by mothers.

A systematic review by Hooley et al concluded that low parental education is associated with high risk of dental caries in children aged 0-6 years.[10] In contrast our study shows low parental education (usually also associated with low socioeconomic status) was associated with a smaller number of children with decayed tooth surfaces. It might be associated with lower consumption of processed sugary food and use of fibrous food in their daily life because parents were unable to purchase processed sugary snacks and children had limited consumption of sugary snacks.

Parental socioeconomic status might influence dental caries in children through oral health knowledge and practices.[11] In our study, upper class and upper middle-class children were having more tooth decayed surfaces as compared to middle class, lower middle class and lower class. It might be due to the busy schedule of upper/upper middle class parents or due to the fact that economically empowered parents always try to fulfill the demands of their children regarding candy, chocolates, snacks, and fast food etc. as they feel guilty having less time to spend with their children due to their busy schedule of life/service/ business/work. Therefore, a higher prevalence of decayed surfaces among children of upper and middle social class was observed in our study while a lower prevalence of decayed surfaces was found among children of lower social class. These findings are similar to the trends reported by researchers of other developing countries.[12-14] India is also a developing country. 
Therefore, our findings are in accordance with the studies mentioned above.

Snacks are considered to be cariogenic. Percentage of decayed tooth surfaces was highest in those children who consumed both sugary and non-sugary snacks followed by sugary snacks only and non-sugary snacks only. No significant association found between decayed primary tooth surfaces and consumed snacks.

Diet plays an important role in the development of caries. The children who consumed both vegetarian and non-vegetarian diet had less percentage of decayed surfaces than those who consumed only vegetarian diet. This might be due to texture, fibrous nature, consistency, adhesiveness, and sugary content of food and other factors like oral hygiene, positions of tooth in the dental arch etc.

Breakfast consumption trends are common in an individual's daily life. Nature of breakfast plays a crucial role in development of cariously decayed surfaces in preschool children. The breakfast variables and decayed surfaces had close correlation in our study. Among the study population decayed tooth surfaces were present in $67.7 \%$ of those children who had history of consuming breakfast in the form of roti with oil and salt, and in $24 \%$ of those children who had history of having breakfast like tea and Paratha. Decayed surfaces were noted in only $8.3 \%$ of those children who had history of consuming breakfast like cheese and bread/butter and bread with milk or tea. It might be due to increased fat content of breakfast. But no statistically significant correlation observed between decayed primary tooth surfaces and breakfast variables $(p=0.719)$. Several studies reported a significant correlation of caries with frequent exposure to sugary food, $[15,16]$ while some studies found weak relationships with dental caries. [17,18]

Feeding habit affects development of decayed surfaces in preschool children. Decayed surfaces were observed in $67.7 \%$ of those children who were breast fed and in $32.3 \%$ of those children who were both breast and bottle fed. This decrease in decayed tooth surface percentage might be related to texture of artificial nipple and breast nipple.

Duration of breast feeding also affects development of decayed surfaces in preschool children. The percentages of decayed surfaces gradually increased up to duration of 1 year then abruptly a high percentage of decayed surfaces were observed. Duration of breastfeeding habit had a significant $(\mathrm{p}=0.001)$ correlation with decay percentage of primary tooth. In children who had history of longer duration of breast feeding, decayed tooth percentage observed was higher. Therefore, breast feeding for longer a duration of time is considered to be harmful to primary tooth.

As the duration of bottle feeding (day and night) increased, percentage of decayed tooth surfaces were also increased. The percentage of children with decayed tooth surfaces gradually increased up to two years of bottle-feeding duration and after that abruptly increased. Longer duration of bottle-feeding habits had high percentage of tooth decayed, as observed in the study. It might be due to low oral clearance rate at night and the fact that oral cavity acts as a closed compartment full of microorganisms. It might favor putrefaction of food on the tooth surfaces and releases organic acid which further demineralizes tooth surfaces. Thus, a significant $(\mathrm{p}=0.000)$ association was observed between percentage of decayed tooth surfaces and duration of bottle feeding. These results were similar to the finding of Hattab et al [8] and Dini et al [19]

\section{Conclusion}

1. Age and gender of preschool children had no significant effect on the development of decayed surfaces of primary teeth.

2. Paternal education level does not significantly affect the development of decayed surfaces, while maternal education level had significant effects on the development of decayed surfaces.

3. Prasad's socioeconomic classifications has a significant effect on the development of decayed surfaces. A higher percentage of children from upper and upper middle-class families have decayed surfaces as compared to middle, lower middle and lower class.

4. Types of snacks, vegetarian or non-vegetarian diet, and type of breakfast had no significant effect on the development of decayed primary tooth surfaces.

5. Methods of feeding and duration of feeding have a significant effect on the development of decayed surfaces in preschool children.

Conflict of Interest: None declared.

\section{References}

1. Prakash H, Sidhu SS, Sundaram KR. Prevalence of dental caries among Delhi school children. J Ind Dent Assoc 1999;70:12-14

2. Reisine S, Litt M. Social and psychological theories and their use for dental practice. Int Dent J 1993;43(3 Suppl 1):279-287

3. Amstutz RD, Rozier RG. Community risk indicators for dental caries in school children: an ecologic study. Community Dent Oral Epidemiol 1995;23(3):129-137 doi:10.1111/j.1600-0528.1995. tb00216.x 
4. WHO. Oral health Survey; Basic methods. Edi.4 Geneva :WHO; 1997

5. Pandey VK, Agarwal P, Kakkar R. Modified BG Prasad's Socioeconomic Classification-2018: The need of an update in the present scenario. Indian J Comm Health 2018;30:82-84

6. Srivastava VK. Prevalence and pattern of caries in primary anterior teeth of preschool children: An observational study. J Indian Soc Pedod Prev Dent 2020;38(1):26-33 doi:10.4103/JISPPD. JISPPD_62_20

7. Vignarajah S, Williams GA. Prevalence of dental caries and enamel defects in the primary dentition of Antiguan pre-school children aged 3-4 years including an assessment of their habits. Community Dent Health 1992;9(4):349-360

8. Hattab FN, Al-Omari MA, Angmar-Månsson B, Daoud N. The prevalence of nursing caries in one-to-four-year-old children in Jordan. ASDC J Dent Child 1999;66(1):53-58

9. Al-Hosani E, Rugg-Gunn A. Combination of low parental educational attainment and high parental income related to high caries experience in pre-school children in Abu Dhabi. Community Dent Oral Epidemiol 1998;26(1):31-36 doi:10.1111/j.1600-0528.1998. tb01921.x

10. Hooley M, Skouteris H, Boganin C, Satur J, Kilpatrick N. Parental influence and the development of dental caries in children aged 0-6 years: a systematic review of the literature. J Dent 2012;40(11):873-885 doi:10.1016/j.jdent.2012.07.013

11. Qui RM, Lo EC, Zhi QH, Zhou Y, Tao Y, Lin HC. Factors related to children's caries: a structural equation modeling approach. BMC Public Health 2014; 14:1071
12. Addo-Yobo C, Williams SA, Curzon ME. Dental caries experience in Ghana among 12-year-old urban and rural schoolchildren. Caries Res 1991;25(4):311-314 doi:10.1159/000261382

13. Nörmark S. Social indicators of dental caries among Sierra Leonean schoolchildren. Scand J Dent Res 1993;101(3):121-129 doi:10.1111/j.1600-0722.1993.tb01650.x

14. Cleaton-Jones P, Chosack A, Hargreaves JA, Fatti LP. Dental caries and social factors in 12-year-old South African children. Community Dent Oral Epidemiol 1994;22(1):25-29 doi:10.1111/j.1600-0528.1994.tb01564.x

15. Kalsbeek H, Verrips GH. Consumption of sweet snacks and caries experience of primary school children. Caries Res 1994;28(6):477-483 doi:10.1159/000262023

16. Akyuz S, Pince S, Garibagaoglu M. Nutrient intake and dental health in school children. J Marmara Univ Dent Fac 1996;2(23):535-539

17. Schröder U, Granath L. Dietary habits and oral hygiene as predictors of caries in 3-year-old children. Community Dent Oral Epidemiol 1983;11(5):308-311 doi:10.1111/j.1600-0528.1983. tb01899.x

18. Burt BA, Eklund SA, Morgan KJ, Larkin FE, Guire KE, Brown LO, et al. The effects of sugars intake and frequency of ingestion on dental caries increment in a three-year longitudinal study. J Dent Res 1988;67(11):1422-1429

19. Dini EL, Holt RD, Bedi R. Caries and its association with infant feeding and oral health-related behaviours in 3-4-yearold Brazilian children. Community Dent Oral Epidemiol 2000;28(4):241-248 doi:10.1034/j.1600-0528.2000.280401.x 